\title{
Effective luminance contrast as a parameter in contingent aftereffects
}

\author{
H. H. MIKAELIAN \\ University of New Brunswick, Fredericton, New Brunswick E3B 5A3, Canada
}

\begin{abstract}
Variation in the magnitude of contingent aftereffects was explored as a function of the luminance contrast ratio profile of inspection stimuli. Positive contrast stimuli (chromatic bars interspersed with achromatically brighter bars) produced substantially attenuated aftereffects compared with negative contrast stimuli (chromatic and dark bars). This attenuation was hypothesized to result from retinal image deterioration of positive contrast gratings due to retinal reflection/scatter. In a second experiment, subjects selected for their light retinal pigmentation manifested substantial enhancement of the asymmetry between the aftereffects generated by positive and negative contrast gratings. Those with dark fundus pigmentation showed symmetrical aftereffects.
\end{abstract}

Chromatic aftereffects that are orientation specific, popularly known as McCollough effects, are normally generated by alternate, and prolonged, viewing of complementary chromatic stripes in orthogonal orientations, such as red vertical stripes alternating with green horizontal stripes; the aftereffects appear as desaturated hues on achromatic stripes, in this case a greenish hue on verticals and a pinkish hue when these are oriented horizontally (McCollough, 1965). The most common explanation of this phenomenon has been in terms of chromatic adaptation of cortical edge detector units that are known to be orientation specific, although Harris and Gibson (1968) have suggested a simpler "dipole" model. A more traditional "learning" type of explanation has also been suggested (Murch, 1976).

The inspection stimuli used to generate these aftereffects normally consist of high-contrast gratings projected through gelatin filters of various band pass characteristics, with the result that they appear to the subject as chromatic (red) bars interspersed with black bars in one orientation and as chromatic (green) bars interspersed with black bars in the orthogonal orientation. In such a configuration, the stripes are differentiated not only by their hue differences (green or red against black), but also by their luminance differences, the black bars being less bright. It is reasonable to assume that both of these features of the inducing stimuli (chromatic contrast and luminance contrast) play important roles in the generation of the contingent chromatic aftereffects,

Reprint requests should be addressed to the author, Department of Psychology, University of New Brunswick, Box 4400 , Fredericton, New Brunswick E3B 5A3, Canada. This research was supported by Grant A0289 from the National Research Council of Canada. A preliminary report on this experiment was presented at the meeting of the Eastern Psychological Association, Boston, 1977. as is the case in many other visual phenomena entailing color and form processing (Graham, 1965). Ellis (1977) has investigated luminance contrast of inspection stimuli as a parameter in the production of McCollough effects. His results show that reducing luminance contrast of these stimuli yields a reduction in aftereffect magnitude, and that the relationship between aftereffect magnitude and changes in luminance contrast is linear. Ellis (1977) used inspection gratings at six different contrast ratios $(.46, .31$, .19 , and .00 ). The various contrast ratio gratings were produced by projecting slides of achromatic gratings of different contrast ratios through gelatin filters, with space averaged luminance adjusted by N.D. filters. While such a procedure is adequate for producing higher contrast ratio gratings, it has an undesirable consequence at lower ratios in that it tends to generate gratings that are visibly tinted throughout; thus, instead of producing gratings consisting of chromatic bars interspersed with achromatic bars, it generates chromatic bars juxtaposed with chromatic bars of the same hue but of lower chromatic value. How the .00 contrast level was achieved is not clear, but, judging from the description, it must have consisted of projecting a blank slide through the gelatin filters. The net result of such a procedure, especially at low-contrast ratios, is to reduce both luminance and chromatic contrast simultaneously, thus making it difficult to evaluate the role of luminance contrast independently.

An additional aspect of the Ellis (1977) study was that the contrast profile used, namely chromatic bars interspersed with other bars of lower luminance, represented only one half of the inspection stimulus luminance profiles possible, the other half being chromatic bars interspersed with achromatic bars of higher luminance. His method of stimulus generation, of course, precluded this possibility. The fol- 
lowing study was designed to explore systematically, and without the restrictions of the Ellis (1977) procedure, variations in the magnitude of the McCollough aftereffects when these are induced by stimuli whose brightness contrast profiles vary from very high (red/ black and green/black or red/white and green/white bars) down to zero (isoluminance), where the edges are defined purely by hue differences (red/gray and green/gray bars of equal luminance).

\section{EXPERIMENT 1}

\section{Method}

Subjects. Sixty undergraduates with no previous experience with McCollough effects and with normal color vision, as tested by American Optical pseudoisochromatic plates, served as subjects.

Apparatus. The inspection stimuli were prepared as follows. Green and red Lettrafilm matte sheets (Pantone 347D, green; and $185 \mathrm{D}$, red) were placed over white posterboards cut to fit the holders of a Scientific Prototype two-channel tachistoscope (the Lettrafilm sheets have an adhesive back, which greatly simplifies construction). Different values of gray Lettrafilm sheets were made by sandwiching various gray and white Lettrafilm matte sheets together (these sheets are weakly translucent). The specific mixtures of the matte sheets for the desired grays were determined previously by obtaining photometric (photo research spor photometer) measures of the different mixtures of Lettrafilm sheets in the tachistoscope (GE, F6.15.D/HH Daylight Fluorescent). Each custom-made gray value (which varied from white to very dark gray, corresponding to Munsell gray values 9.0 to 2.75) was cut into narrow, 2-mm-wide strips (using a rotary blade photographic film trimmer) and pressed on the green and red sheets at 4-mm intervals, thereby forming stripes consisting of chromatic bars interspersed with achromatic bars. It was thus possible to construct inspection stimuli whose luminance contrast profile$[(L \max -L \min ) /(L \max +L \min )] \times 100$-varied from $-60 \%$ (chromatic bars interspersed with darkest gray bars) to $+60 \%$ (chromatic bars interspersed with white bars). All the custom-made grays as well as the red and green sheets used in constructing the stimuli were matched, in the tachistoscope, against Munsell chips to obtain their Munsell equivalents (red $=5 R, 5 / 14$; green $=2.5 \mathrm{G}, 5 / 12$ ) . This was done to provide, for the colors used, a reference to standard colors. The reason that Munsell colors were not used in constructing the stimuli in the first place was the relative difficulty of preparing the inspection stimuli (24 in all) using sheets that did not have an adhesive backing. An additional fact to be noted is that the photometrically measured luminance contrast ratios of the inspection stimuli at the highest positive and negative contrast levels $(-60 \%$ and $60 \%)$ are slightly different (between $5 \%$ and $7 \%$ ) from the calculated contrast ratios of their equivalent Munsell colors (using tabulated percent reflectances); this is probably due to the spectral characteristics of the illuminant and to the nature of the Lettrasette pigment. The specific luminance contrast ratios used were: $-60 \%,-30 \%, 0 \%, 20 \%, 30 \%$, and $60 \%$; four stimuli were constructed at each contrast level (horizontal and vertical red gratings, horizontal and vertical green gratings). The spatial frequency of the stimuli when viewed in the tachistoscope was 3.4 cycles $/ \mathrm{deg}$, with a visual field of $5 \times 5 \mathrm{deg}$. Space average luminance was adjusted separately in each channel of the tachistoscope (by varying the intensity of the illuminant) to a fixed value of $15 \mathrm{~cd} / \mathrm{m}^{2}$, thereby making it identical for all the inspection gratings used (White, 1976). [This luminance value might seem somewhat low, but it was the best that could be achieved within the tachistoscope, given the reflection characteristics of the stimuli. White (1976) also reports being able to obtain aftereffects at equivalent luminance levels.]

The magnitude of the aftereffect was measured by using a nulling technique described by Riggs, White, and Eimas (1974). Briefly, it consisted of adding red and green light to the horizontal and vertical test grating (illuminated with achromatic light) until the subject reported a color match (or no color difference) between the orthogonal gratings. The amount of red and green added was converted into measures of excitation purity $\left(\mathrm{P}_{\mathrm{e}}\right)$ and used as an estimate of aftereffect strength. The test grating on which aftereffect measures were taken consisted of adjacent horizontal and vertical gratings with a spatial frequency of $3.4 \mathrm{cycles} / \mathrm{deg}$, a brightness contrast ratio of $60 \%$, and a space average luminance of $41 \mathrm{~cd} / \mathrm{m}^{2}$. The red and green filters in the test apparatus were Wratten No. 25 and No. 58 , respectively, and the projection light used was a tungsten light source (CLMB).

Procedure. The 60 subjects were divided randomly into six groups of 10 each, one group for each inspection stimulus contrast level. Each subject participated in the experiment in two separate sessions and viewed the inspection stimuli at only one contrast level. The sessions differed according to the colororientation contingencies of the inducing bars. In Session 1, the red inducing bars were vertical and the green horizontal, while in Session 2 , the contingencies were reversed. Sessions 1 and 2 were separated by at least $48 \mathrm{~h}$, with half of the subjects being exposed to Session 1 first and the other half to Session 2 first.

The between-subjects design used in this experiment was dictated by the long decay time of the McCollough effects. Not only do these effects persist, but they also interact with subsequent exposure; for instance, following apparent decay, one can readily revive the aftereffect by very short reexposure periods. Although aftereffect magnitude is measured as the difference between pre- and postexposure adjustments (a procedure used to reduce contamination from persistent effects), it is not readily possible to avoid the interaction of previous exposure on a subsequent one (except by using very long interexposure intervals). Since the design of this experiment called for repeated exposure to inducing stimuli that varied only in luminance contrast, the between-subjects design seemed the most appropriate. The sessions variable was retained as a within-subjects variable because it is much less susceptible to contamination.

The procedure for each session was as follows: After pretraining on the use of the test apparatus with several practice trials (turning a hand knob allowed the subject to vary, through a servo system, the amount of red and green to be added to the vertical and horizontal test grating to achieve a null), six preexposure measures of the subjective null were obtained. The subject was then instructed to view through the tachistoscope, which presented the inducing red and green gratings in alternation at 8-sec intervals. Total exposure lasted for $20 \mathrm{~min}$, following which six postexposure measures of the subjective null were again obtained. Differences between pre- and postexposure measures in excitation purity $\left(\Delta \mathrm{P}_{\mathrm{e}}\right)$ were used as indications of the strengths of the chromatic aftereffects.

\section{Results}

The results are shown in Figure 1. The ordinate represents aftereffect magnitude in mean $\Delta \mathrm{P}_{\mathrm{e}}$, and the bars are standard errors. Since there were no significant differences between Session 1 and Session 2 data, they were combined. The data clearly show that the procedure used does, indeed, generate reliable contingent chromatic aftereffects and that the magnitude of the aftereffect varies significantly as a function of the luminance contrast ratio profile of the inducing stimuli $[F(5,54)=6.16, p<.001]$. High negative contrast stimuli $(-60 \%$, chromatic bars interspersed with dark bars) generate the largest effect, which gradually declines with decreasing contrast ratios, reaching a minimum at $20 \%$. At $60 \%$ contrast (chromatic bars interspersed with white bars), after- 


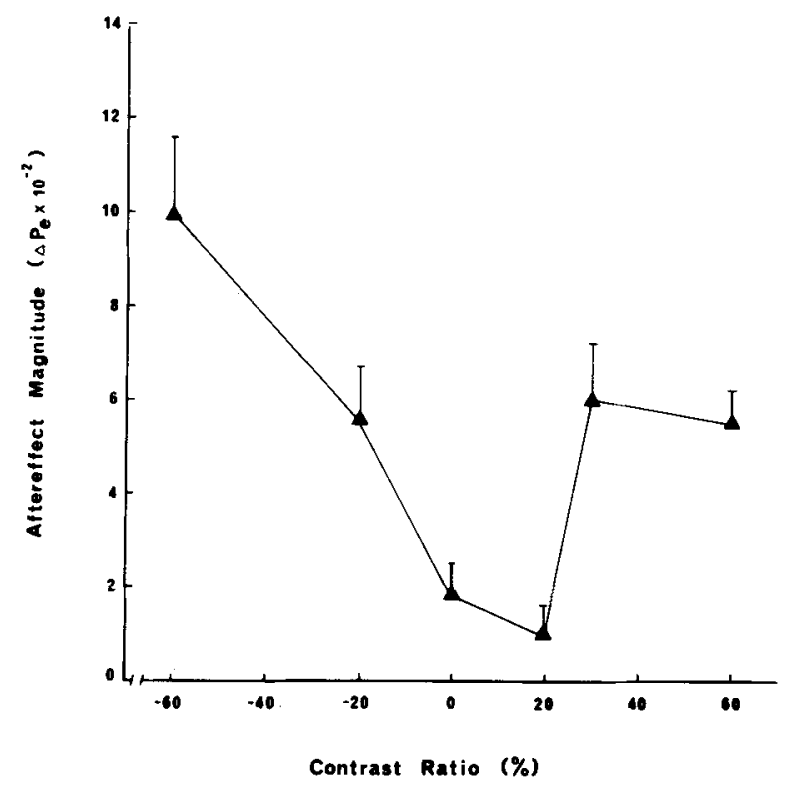

Figure 1. Magnitude of contingent chromatic aftereffects as a function of luminance contrast ratio of inspection stimuli. Data points are mean $\Delta P_{e}$; the bars are $1 / 2$ standard errors in length.

effect magnitude is smaller than at $-60 \%$ [t(18) $=3.042, \mathrm{p}<.01]$ and no different from that at $30 \%$ or $-20 \%$.

\section{Discussion}

The data clearly show that the generation of strong McCollough effects requires high-contrast inspection stimuli and, further, that aftereffects are stronger when the inducing chromatic edges are outlined by achromatically darker bars. The fact that low-contrast ratio adaptation stimuli generate weak effects suggests that luminance contrast is, in addition to orientation and chromaticity, a critical variable in the induction of the McCollough effects; the state of affairs is somewhat complex, however, since, at photometric isoluminance $(0 \%$ contrast $)$, significant effects still appear evident $[t(8)=6.347, p<.01]$, the effect reaching a minimum only at $20 \%$.

The reduction in aftereffect magnitude cannot be ascribed to poor visibility of the gratings-as the inducing bars were clearly visible at all the contrast ratios-and is generally consistent with Gouras' (1972) proposed model of edge information processing related to the generation of McCollough effect. That model stipulates that, in mediating colored edges, chromatic and achromatic (form) information are extracted into separate channels after a stage of orientation selectivity; when achromatic information is substantially reduced (as is the case at very lowcontrast ratios), input necessary for the determination of orientation specificity is also reduced, thereby attenuating any orientation-specific chromatic aftereffects.
The asymmetry in aftereffect magnitude obtained with negative- and positive-contrast inducing stimuli is puzzling, but consistent with previously reported data (Harris \& Barkow, 1969). While there may be several explanations that could account for this asymmetry, one that involves only peripheral factors seems particularly expedient. The difference in the magnitude of aftereffects between positive- and negative-contrast inspection stimuli may simply be due to the fact that the negative contrast edges, being circumscribed by adjacent chromatic and dark regions, are better defined on the retina (Walls, 1943), due to reduced reflection and scatter of light at the fundus, and thus produce stronger aftereffects.

The possibility that differences in the clarity of edge definition on the retina (due to reflection/scatter of light at the fundus as well as other factors) may have a bearing on the generation of McCollough effects leads to interesting consequences. It is a common observation that while some subjects appear to be susceptible to McCollough effects (with the usual high-contrast inducing gratings), others are less so, a situation generally ascribed to "individual variability." The present experiment was not exempt from such variability; for example, at $0 \%$ and at $20 \%$ contrast, a number of subjects showed small, but reliable, aftereffects, while others failed to show any effects at all. It could be argued that the subjects showing aftereffects at $0 \%$ contrast (photometric isoluminance) were able to do so because, due to characteristics of their fundi, they did not process these edges as being isoluminant. Similarly, subjects showing no aftereffects at $20 \%$ contrast processed those edges as being at effective isoluminance, also due to the characteristics of their ocular media, despite the presence of photometrically measured brightness differences. It is not unreasonable to speculate that differences of as much as $20 \%$ or more could be present for individual subjects' brightness values when luminances are photometrically equal. The implication of such an analysis is, of course, that the variability in McCollough effects among different subjects may, in part, be accounted for by differences in the characteristics of the ocular media of the subjects.

Since the subjects in each contrast ratio condition in the present experiment were exposed only to one condition, it was not possible to verify one obvious consequence of the speculation, which is that those showing no aftereffect at $0 \%$ show reliable aftereffects at $20 \%$ contrast levels, the reverse being true for those who show reliable aftereffects at $0 \%$. There is, however, an alternative, and more direct, way of investigating the role of retinal reflection/scatter characteristics in the generation of McCollough effects. Fundus pigmentation is an index that is, perhaps most directly, related to this characteristic; it should be possible, then, to explore the above hypothesis by 
separating groups according to their fundus pigmentation and inducing the contingent chromatic aftereffects by the various contrast ratio inspection stimuli. The following experiment was designed to carry out such a procedure.

\section{EXPERIMENT 2}

\begin{abstract}
Method
Subjects and selection procedure. Forty-eight new subjects, 24 with lightly pigmented fundi and 24 with darkly pigmented fundi, were selected. All subjects had normal color vision, as tested by American Optical pseudoisochromatic plates, and had normal or corrected vision. Fundus pigmentation was determined as follows: Optical density of the ocular medium was measured by determining retinal reflectance using a modified version of the Campbell and Rushton (1955) optical system. Briefly, the operation consisted of projecting the image of a 1-deg-diam light source of known radiometric intensity into the subject's retina (6 deg lateral to the fovea) and measuring the intensity of the reflected light with a photodetector mounted on one channel of a binocular indirect ophthalmoscope. Four different filters in the path of the light source were used: Wratten No. 58 (green), No. 48 (blue), No. 25 (red), and No. 15 (yellow). During measurement, the subject viewed a fixation point with the unexamined (left) eye, with his/her head stabilized with a biteboard mounted on a motorized $\mathrm{X}-\mathrm{Y}$ mover; the observer could adjust the position of the subject's eye quite precisely by viewing it through one of the channels of the binocular ophthalmoscope. Subjects were dark adapted for $20 \mathrm{~min}$ before measurement, and each measurement was repeated four times (during the same session). Optical density for each filtered measurement was calculated for each subject. If the density measures were below an arbitrarily determined figure, the subject was categorized as possessing lightly pigmented fundi; if they were above that figure, he was categorized as having darkly pigmented fundi. Quite commonly the measures straddled the dividing line, and those subjects were disqualified. This strategy was adhered to so as to enhance the difference in fundus pigmentation between the two groups of subjects. In general, density measures obtained with filters $15\left(\lambda_{d}=585.9\right)$ and $24\left(\lambda_{d}=612\right)$ were not useful in discriminating between the light- and darkfundus subjects (both yielded low-density figures for all subjects). Measures using filters $47\left(\lambda_{d}=470.1\right)$ and $58\left(\lambda_{d}=538.2\right)$ allowed more reliable discrimination. Mean density measures are shown in Table 1.

While the procedure used above in arriving at a decision on the fundus pigmentation of subjects is not without its problems and shortcomings, especially since the optical density measures represent the characteristics of the entire ocular medium and not just of the retina, it is a relatively satisfactory way of placing into the light-fundus-pigmentation group subjects whose fundus pigmentation is quite light, and into the darkly pigmented fundus group those subjects with highly pigmented retinas.

Some comments on the problem of subject selection would be
\end{abstract}

Table 1

Mean Optical Density Measures of the Three Groups of Subjects

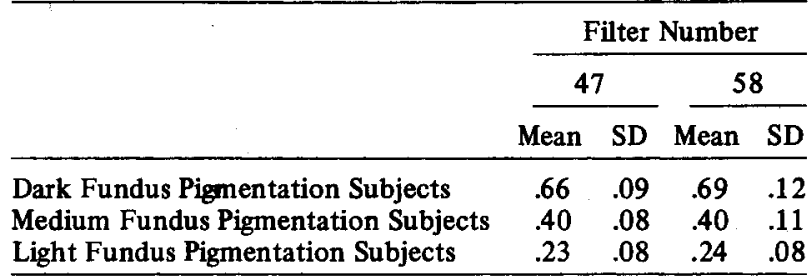

Note-Medium fundus pigmentation subjects were excused. appropriate here. To enhance the hit rate for subjects with sufficiently light fundus pigmentation, mostly subjects with lightly pigmented irises were screened for their fundus pigmentation; similarly, for the dark fundus pigmentation group, subjects with dark iris pigmentation were screened. In the latter group were included a number of Nigerian students; it should be pointed out, however, that nearly $60 \%$ of these subjects did not possess sufficiently dark fundus pigmentation. In the overall group, only $60 \%$ (14 out of 24) were black students; the remainder consisted of Caucasian students from the Province of New Brunswick. In the light-funduspigmentation group, all but one were Caucasians from the Province; the one exception was a Nigerian student. In general, it was much easier to find subjects in the lightly pigmented group than those who could qualify for the darkly pigmented group, a situation that may have been due partly to the selection criterion and partly to the population of the region.

Procedure. The subjects in each of the two fundus-pigmentation groups were divided randomly into six subgroups, each subgroup being assigned to one of the six inspection stimulus contrast ratio conditions $(-60 \%,-30 \%, 0 \%, 20 \%, 30 \%$, and $60 \%)$. Each of the six subgroups thus consisted of four subjects with lightly pigmented fundi and four with darkly pigmented fundi. Exposure and test procedures were as in Experiment 1. A balanced design was used in which the subjects in each subgroup were exposed to the appropriate inducing stimuli in two separate sessions; the sessions, as in Experiment 1, differed on the basis of the color/ orientation contingencies of the inspection gratings.

\section{Results}

The results are shown in Figure 2, which includes the data from Experiment 1 for comparison (dashed line). As before, the data from Sessions 1 and 2 were combined. Reliable effects for the darkly pigmented fundus group $[F(5,18)=9.08, p<.0001]$ are obtained at all contrast ratios except at $0 \%$; for the lightly pigmented fundus group $[F(5,18)=11.87$, $\mathrm{p}<.0001$, the corresponding point is $20 \%$.

\section{Discussion}

The results show that the functions for the two groups of subjects are different. For the darkly pigmented fundus group, the curve appears to be almost symmetrical about the origin. Unlike the previous results, no reliable differences in the magnitude of the aftereffects exist between positive- and negativecontrast ratio conditions, and at $0 \%$ contrast, when the inspection bars are at photometric isoluminance, no contingent chromatic aftereffects are produced.

The data from the lightly pigmented fundus group, however, demonstrate most of the asymmetries observed in Experiment 1. Not only are the aftereffects larger with negative-contrast adaptation stimuli (chromatic bars interspersed with dark bars), but also significant aftereffects are still produced at the $0 \%$ contrast level $[\mathrm{t}(3)=6.44, \mathrm{p}<.01]$; it is only at $20 \%$ that these are reduced to zero. Additionally, there is a reversal of the difference in aftereffect magnitude between the two groups as a function of positive vs. negative contrast; with negative-contrast inspection stimuli ( $-60 \%$ and $-20 \%)$, the aftereffects manifested by the lightly pigmented fundus group are significantly larger than those of the darkly pigmented fundus group $[t(14)=3.64, p<.01]$; the situation is reversed 


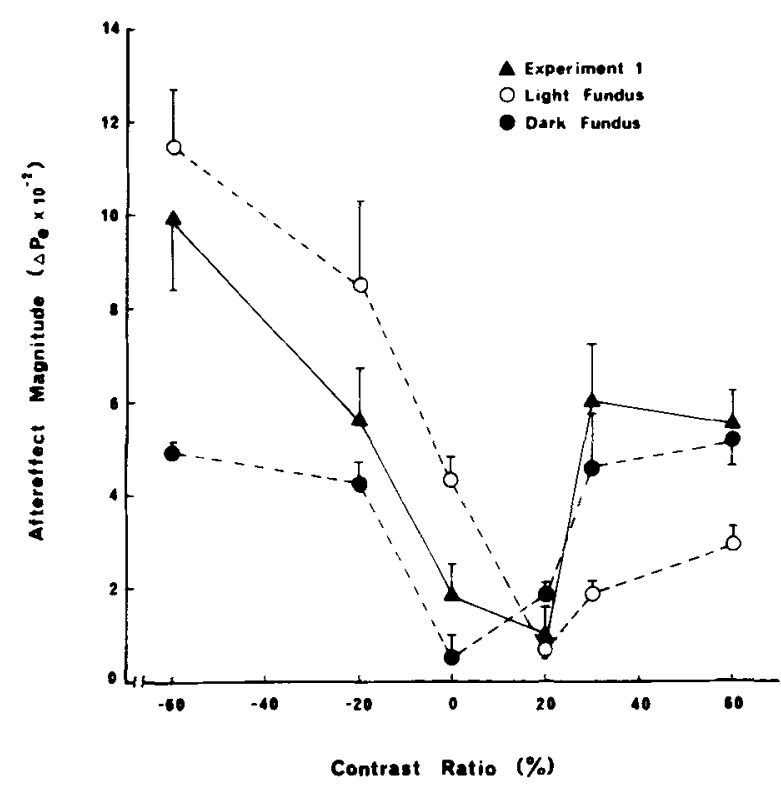

Figure 2. Variation in the magnitude of contingent aftereffects as a function of luminance contrast of inspection stimuli and fundus pigmentation (the dashed line is the data from the first experiment). Data points are mean $\Delta P_{e} ;$ the bars are $1 / 2$ standard errors.

with positive-contrast inspection stimuli $(60 \%$ and $30 \%)[t(14)=2.76, p<.02]$. The data of Experiment 1 fit a reasonably satisfactory set of midpoints of two functions, a situation that is to be expected, since the subjects in Experiment 1 were selected without regard to their fundus pigmentation, thereby yielding a group that probably included a number of subjects with lightly pigmented fundi as well as several with dark fundus pigmentation.

These data are consistent with the speculation discussed at the conclusion of Experiment 1, and suggest that the magnitude of the contingent chromatic aftereffects is influenced not only by the photometrically defined luminance profile of the inducing stimuli, but also by the effective luminance contrast of these gratings as mediated by fundus pigmentation. There could be several ways in which fundus pigmentation could be modulating the contrast of the inducing gratings. Considering, for the moment, a lightly pigmented retina, the image of a $0 \%$ luminance contrast ratio, say of red and gray gratings, results in considerable reflection of the red (from the pigmented layers posterior to the photoreceptors), thereby changing the effective luminance contrast ratio from isoluminance into some negative value (as defined in this experiment). Such a situation will be conducive to the production of aftereffects, since now the inspection stimulus possesses an effective luminance differential (for the achromatic channel) and a color difference (for the chromatic channel). At $20 \%$ contrast, however, where the neutral bar is photometrically brighter than the chromatic bar, dif- ferential reflection favoring the red could reduce the luminance difference between the red and gray gratings, thereby producing an effective luminance contrast ratio that might be near $0 \%$; under such a condition, aftereffects should be substantially attenuated, and that is the case. This analysis may also be extended to the other negative-contrast ratios as well; the photometrically defined $-20 \%$ and $-60 \%$ contrast ratios may, for the lightly pigmented retinas, possess effective contrast ratios of more than $-20 \%$ and $-60 \%$, which could thus account for the larger aftereffects.

The situation is somewhat more complex for the positive-contrast ratio data. The lightly pigmented retina will produce not only significant reflection, thereby reducing the luminance difference between the chromatic and white bars, but also significant scatter from both the chromatic and the achromatic (now more luminant) regions of the retinal image, thereby adding to the deterioration in the quality of edge definition of the retina. The combination of these two factors, viz, reduction in effective contrast ratio and image deterioration due to light scatter at the retina, could account for the substantial depression in the magnitude of the aftereffect.

For the darkly pigmented fundus group, aftereffect magnitudes for positive-contrast ratios do not differ from those obtained at negative ratios. Since these subjects were selected for very dark fundus pigmentation, it is quite likely that reflection and scatter of light at their retinas were relatively minimal, thereby producing little, if any, deterioration in the quality of edge definition on the retina or any change in the photometrically defined luminance ratios.

These arguments are presented as tentative explanations for the present data and, of course, remain to be verified. While the results do not directly bear on the cortical edge detector theory of the McCollough effects, they do tend to argue against a "learning" interpretation of the phenomenon; perceptually, the inspection gratings at all the luminance contrast ratio conditions were clearly visible and should have thus produced aftereffects at all contrast levels.

The results of the present experiments and the proposed explanation do point to a number of interesting experiments and predictions; for instance, since scatter of light at the retina affects low spatial frequencies less than high frequencies (Westheimer \& Campbell, 1962), it should be possible to demonstrate that functions that relate aftereffect magnitude to spatial frequencies of inducing stimuli would vary, depending upon fundus pigmentation. One can, of course, ask the same questions about aftereffects induced by blue and yellow adaptation gratings, since, after all, it is in the perception of these colors that fundus pigmentation is a more critical variable (Ebert \& Pollack, 1972; Ishak, 1952a, 1952b; Ruddock, 
1963; Wald, 1949; Wright, 1928). These experiments are currently being implemented and will be reported later.

\section{REFERENCES}

Campbell, F. W., \& Rushton, W. A. H. Measurement of the scotopic pigment in the living human eye. Journal of Physiology, 1955, 130, 131-147.

Ebert, P. C., \& Pollack, R. H. Magnitude of the MuellerLyer illusion as a function of lightness contrast, viewing time, and fundus pigmentation. Psychonomic Science, 1972, 26, $347-348$.

ELLIS, S. R. Orientation specificity of the McCollough effect: Analysis by equivalent contrast transformation. Perception \& Psychophysics, 1977, 22, 539-544.

Gouras, P. Color opponency from fovea to striate cortex. Investigative Ophthalmology, 1972, 11, 431-437.

GraHam, C. H. Vision and visual perception. New York: Wiley, 1965.

Harris, C. S., \& Barkow, B. Color/white grids produce weaker orientation-specific color aftereffects than do color/black grids. Psychonomic Science, 1969, 17, 123. (Abstract)

Harris, C. S., \& Gibson, A. R. Is orientation-specific color adaptation in human vision due to edge detectors, afterimages or "dipoles"? Science, 1968, 162, 1506-1507.

IsHak, I. G. H. The photopic luminosity curve for a group of fifteen Egyptian trichromats. Journal of the Optical Society of America, 1952, 42, 529-534. (a)
IshaK, I. G. H. The spectral chromaticity coordinates for one British and eight Egyptian trichromats. Journal of the Optical Society of America, 1952, 42, 534-539. (b)

McCollough, C. Color adaptation of edge detectors in the human visual systems. Science, 1965, 149, 1105-1106.

Murch, G. M. Classical conditioning of the McCollough effect: Temporal parameters. Vision Research, 1976, 16, 615-619.

Riggs, L. A., White, K. D., \& Eimas, P. D. Establishment and decay of orientation-contingent aftereffects of color. Perception \& Psychophysics, 1974, 16, 535-542.

RuDDock, K. H. Evidence for macular pigmentation from colour matching data. Vision Research, 1963, 3, 417-429.

WALD, G. The photochemistry of vision. Documenta Ophthalmologica, 1949, 3, 94-137.

WALLS, G. L. Factors in human visual resolution. Journal of the Optical Society of America, 1943, 33, 487-505.

Westheimer, G., \& Campbeli, F. W. Light distribution in the image formed by the living human eye. Journal of the Optical Society of America, 1962, 52, 1040-1045.

WhITE, K. D. Luminance as a parameter in the development and testing of McCollough effects. Vision Research, 1976, 15, 297-302.

WRIGHT, W. D. A re-determination of the trichromatic coefficients of the spectral colors. Transactions of the Optical Society (London), 1928, 30, 141-158.

(Received for publication October 29, 1979; revision accepted January 2, 1980.) 